\title{
Short-term visual storage
}

STEVEN W. KEELE 2 AND WILLIAM G, CHASE

UNIVERSITY QF WISCONSIN

The delay between the offset of a briefly exposed array of letters and digits and the onset of an arrow pointing at one of the array positions was varied from 0 to $5000 \mathrm{msec}$. In addition, the luminance of the stimulus array was varied over three levels. The Ss reported the item in the position indicated by the arrow. Luminance, delay, and the luminance by delay interaction were all significant, Performance monotonically decreased from a delay of $0 \mathrm{msec}$ to a delay of $250 \mathrm{msec}$, but the percent correct remained fairly constant from $250 \mathrm{msec}$ to $5000 \mathrm{msec}$. With delays shorter than 250 msec, high luminance arrays showed better performance.

A series of experiments by Sperling (1960) and by Averbach and Coriell (1961) suggested a short-term visual store. Their tasks involved brief tachistoscopic exposures of information followed after a variable interval by some indicator signalling $S$ to report a specific part of the information. Sperling presented his Ss with two or three rows of letters for $50 \mathrm{msec}$ followed at various intervals by one of two or three tones indicating which row to report. He found that over delays between the array and tone ranging from 0 to $500 \mathrm{msec}$, report accuracy fell from about 9.1 items to memory span accuracy of about 4.3 items. The memory span was determined by asking the $S$ to report all the items he could regardless of the row they occurred in. Thus, with the probe technique, Sperling was able to demonstrate that Ss have much more information available than can be reported by the memory span technique. Since this information is available only for about $1 / 2 \mathrm{sec}$, the memory span technique is too slow to demonstrate this storage. Averbach and Coriell (1961) in a similar experiment using a visual marker found the same sort of decay function.

In a recent study by Eriksen and Steffy (1964), a circular arrangement of six Xs and Os was presented briefly and followed after a delay by an arrow pointing at one of the six positions. They found no evidence for a decline in the number of correct reports as the delay between the stimulus array and the indicator increased from 10 to $700 \mathrm{msec}$. Consequently, they questioned the concept of a short-term visual store.

There are at least two possibilities for the apparent discrepancy between the results of Eriksen and Steffy (1964) and those of Sperling (1960) and Averbach and Coriell (1961): (a) Sperling varied the number of stimulus items from three to 12. There was no decline in the percent correct when a partial report technique was used unless the number of items exceeded the memory span. Thus, there is no reason to expect that Eriksen and Steffy would find a decline with six items, especially when it is considered that binary items have a slightly larger memory span than a population including all the letters of the alphabet (Miller, 1956). (b) As pointed out by Eriksen and Steffy, the intensity of their stimulus arrays (3.7 $\mathrm{ft}-\mathrm{L}$ ) was much less than that of Averbach and Coriell $(70 \mathrm{ft}-\mathrm{L})$. It may be that the amount of information in store and the rate of decline of the amount in store is a function of stimulus intensity.

Preliminary investigations indicated that with a 6-item binary array, Ss could greatly reduce the number of positions attended to by using a simple coding strategy. By attending to the positions of one of the symbols, say the Xs, the $\mathrm{S}$ could remember the other positions by elimination. Thus, if the indicator arrow did not point to one of the attended positions, the $\mathrm{S}$ simply reported that the other symbol was indicated even though he had not attended to it. It is therefore apparent that in order to exceed S's memory span, the information content of the arrays must be increased.

The present experiment replicates the main features of Experiments II and III of Eriksen and Steffy (1964). The information content of the arrays is greatly increased, however, by using 10 items rather than six and by using digits and letters rather than Xs and Os. In addition, the luminance of the stimulus array is varied over three levels.

\section{Subjects}

\section{METHOD}

Three women and one man served as paid Ss in this experiment.

\section{Maierials}

Forty-two white stimulus cards were prepared with 10 black letters and digits arranged in a circle. The circle subtended a visual angle of approximately $2.5^{\circ}$ and individual letters and digits subtended a visual angle of approximately $.30^{\circ}$. Each of the 26 letters of the alphabet and the digits 1 through 9 occurred an equal number of times over the set of 42 arrays, but otherwise the items and order of items appearing on a stimulus were randomly determined.

A separate indicator card with a white arrow on a black background was constructed for each of the 10 positions. The arrow originated from a fixation point and pointed at one of the 10 positions. The distance between the head of the arrow and the position at which it was pointed was approximately. $\mathbf{1 2}^{\circ}$. The 
fixation point was a back-lighted pin hole in opaque black poster-board.

The three channels of a Scientific Prototype Tachistoscope were used to present the array, the indicator arrow, and the fixation point.

\section{Design}

On the first day, Ss practiced on the high luminance with a 0 msec delay for 126 trials. On the following days there were three levels of stimulus intensity (3.7, 16, and $70 \mathrm{ft}-\mathrm{L})$ and seven delays between the offset of the stimulus array and the onset of the arrow $(0,50,100,250,500,1000$, and $5000 \mathrm{msec})$. A given delay was run in blocks of six trials and each of the seven delays was run at one level of intensity before shifting to another intensity. The order of the six-trial blocks for delay intervals was randomized for each luminance, and the order of intensities over the nine experimental days was counterbalanced by means of 3 by 3 Latin squares for each $S$. Thus there was a total of 54 trials for each $S$ at each of the 21 delay by luminance conditions.

\section{Procedure}

The Ss viewed the stimuli from a dark chamber that was screened from outside light sources. Prior to beginning each experimental session, Ss were dark adapted in the chamber for 4-5 $\mathrm{min}$.

Upon a signal from $\mathrm{E}$, the $\mathrm{S}$ looked into the tachistoscope, and when he could clearly see the fixation point he initiated a trial by pushing a microswitch. A stimulus array was exposed for $100 \mathrm{msec}$, followed by a blank interval of 0 to $5000 \mathrm{msec}$, and then exposure for $150 \mathrm{msec}$ of an arrow pointing at one of the stimulus positions. The pre-exposure and delay flelds were dark with the exception of the dim fixation point. The Ss were asked to report the letter or digit that the arrow had pointed at. Approximately 10 sec elapsed between report and the following trial.
At the end of 42 trials with one luminance, an independent estimate of the memory span was obtained. Each of five arrays was presented for $100 \mathrm{msec}$, and $S$ reported as many letters as he could.

The Ss knew the delay and luminance conditions in advance but no feedback on the correctness of their responses was given until after all the trials at one luminance.

\section{RESULTS}

The percent correct reports as a function of delay and luminance is plotted in Fig. 1 along with the percentage of the 10 items correctly reported in the memory span condition. For each level of luminance the percent correct shows a monotonic decline from 0 to $250 \mathrm{msec}$. For delays of $250 \mathrm{msec}$ or longer, performance has deteriorated to memory span accuracy of about four items. The amount of decline is greater for the high luminance than for the medium luminance, and the latter shows a greater decline than the low luminance. Large sample approximations of the $95 \%$ confidence intervals for each point in Fig. 1 vary from $\pm 5.7 \%$ to $\pm 6.5 \%$. An analysis of variance showed delay, luminance, and the delay by luminance interaction to be significant $(F=7.92, \mathrm{df}=6 / 18$, error $\mathrm{MS}=$ $.0071, p<.001 ; \mathrm{F}=14.65, \mathrm{df}=2 / 6$, error $\mathrm{MS}=.0123$, $\mathrm{p}<.005$; and $\mathrm{F}=2.21$, $\mathrm{df}=12 / 36$, error $\mathrm{MS}=.0076, \mathrm{p}<$ .05 , respectively).

Figure 2 shows the mean percent correct for Ss as a function of delay interval. Large sample approximations of the $95 \%$ confidence intervals for each point vary from $\pm 5.8 \%$ to $\pm 7.7 \%$. Both Ss 1 and 4 show large effects of delay whereas Ss 2 and 3 show only a slight effect of delay. The marked individual differences in these tasks thus limit the generality of the results.

A further analysis was performed only on the errors. To get enough error data, the errors were pooled for all Ss and all luminances and were further summed over 0,50 , and $100 \mathrm{msec}$ delays (short), 250 and 500

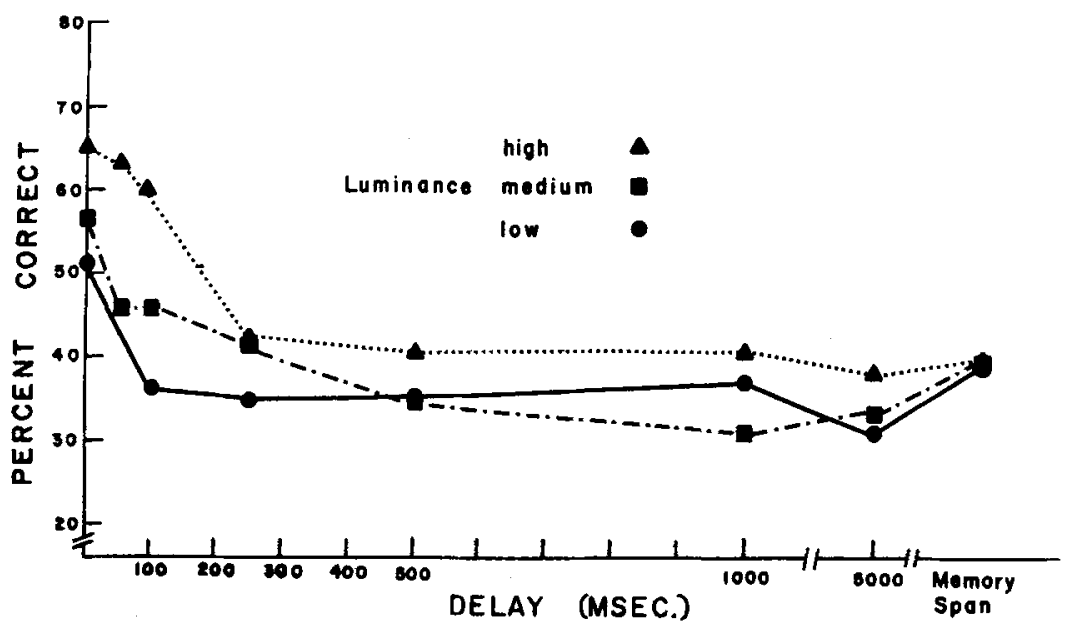

Fig. 1. Mean percent correct reports as a tunction of luminance and delay interval. 


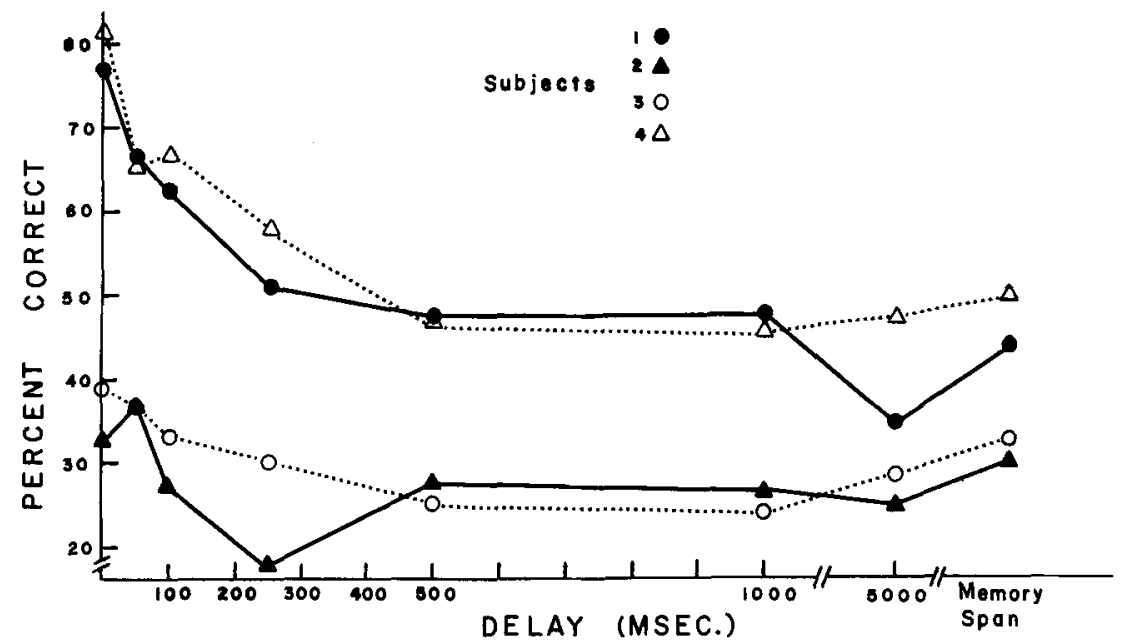

msec delays (medium), and 1000 and 5000 msec delays (long). From the resulting three 35 by 35 stimulusresponse tables were taken the three 26 by 26 submatrices of letter contingency tables. These tables yielded approximately equal error distributions for the three delays: 582,506 , and 534 errors for short, medium, and long delays, respectively. These matrices were then converted to error proportion matrices by dividing the errors for a particular stimulusresponse combination by the number of occurrences of that stimulus. Then the two error proportions for two letters when one was the stimulus and the other the response and vice versa were averaged, resulting in triangular matrices. These confusion matrices were then correlated with a triangular matrix giving the percent overlap in the contours for each possible pair of letters. This percent overlap matrix is a measure of the structural similarity of the letters. The resulting Pearson product-moment correlations were $.37, .17$, and .17 for the short, medium, and long delays, respectively. Two-tailed $z$-tests showed each of the correlations to be significantly greater than zero $(p<.01)$. In addition, the correlation at the short delay was significantly greater than at the longer delays $(p<.01)$.

Similar correlations were obtained between the present confusion matrices and an auditory confusion matrix reported by Conrad (1964). Those correlations were $.02,-.04$, and .09 for the short, medium, and long delays, respectively. None of these correlations were significantly different from zero.

\section{DISCUSSION}

The present experiment demonstrated a large decrease in the accuracy of identification of visually presented information over short delays following the visual exposure. This rapid decline in accuracy was found even with luminance conditions similar to those used by Eriksen and Steffy (1964). It appears, therefore, that their failure to find a similar decline was a result of using a small number of binary items that could be rapidly processed before the information was lost from a short-term visual store.

The visual nature of this storage system is indicated by the fact that Iuminance interacted with delays producing better performance for higher intensity arrays at short delays. In addition, there were more visual confusions at short delays since the correlation between letter confusions and the overlap between letter forms was greater at short delays.

Conrad (1967) has recently shown that errors in the short-term recall of visually presented quadrigrams involved a large number of acoustic confusions. The number of acoustic confusions declined as the retention interval was lengthened over a period of several seconds. This finding is consistent with other recent evidence indicating that acoustic similarity is a potent source of interference in short-term memory tasks (Conrad, 1964; Wickelgren, 1965, 1966; Posner \& Konick, 1966).

The fact that there was no evidence of acoustic confusions in the present experiment suggests that short-term visual storage is a separate system from that studied in short-term memory experiments where retention of material is studied over longer intervals. These findings are consistent with Sperling's (1963) model which postulates two systems: (1) a very brief visual storage system which holds a great deal of information for a short time (up to $1 / 2 \mathrm{sec}$ ) until it can be attended to, and (2) an auditory short-term memory where a limited amount of information is held for several seconds after it has been processed. Visual variables such as visual similarity and luminance would have their effect while material is still in the visual storage system, whereas acoustic and associative variables would have their effect after the information is processed and is in a short-term auditory store or more permanent memory.

\section{References}

Averbach, E., \& Coriell, A. S. Short-term memory in vision. Bell Sys. Tech. J., 1961, 40, 309-328. 
Conrad, R. Acoustic confusions in immediate memory. Brit. J. Psychol., 1964, 55, 75-84.

Conrad, $\mathbf{R}$. Interference or decay over short retention intervals. J. verbal Learn. verbal Behav., 1967, 6, 49-54.

Eriksen, C. W., \& Steffy, R. A. Short-term memory and retroactive interference in visual perception. J. exp. Psychol., 1964, 68, 423-434.

Miller, G. A. The magical number seven, plus or minus two: Some limits on our capacity for processing information. Psychol. Rev., $1956,63,81-97$.

Posner, M. I., \& Konick, A. F. On the role of interference in shortterm retention. $J$. exp. Psychol, 1966, 72, 221-231.

Sperling, G. The information available in brief visual presentations. Psychol. Monogr., 1960, 74, No. 11 (Whole No. 498).
Sperling, G. A modeI for visual memory tasks. Hum. Factors, 1963, 5, 19-31.

Wickelgren, W. Acoustic similarity and retroactive interference in short-term memory. J. verbal Learn. verbal Behav., 1965, 4, 53-61.

Wickelgren, $W$. Phonemic similarity and interference in short-term memory for single letters. J. exp. Psychol., 1966, 71, 396-404.

\section{Notes}

1. This experiment was supported by PHS fellowship to the first author.

2. Now at the University of Oregon.

(Accepted for publication April 28, 1967) 